\section{Laurent Schaeffer Jean-Marc Egly}

\section{ADRESSE}

L. Schaeffer : boursier BDI/Cnrs. J.-M. Egly : directeur de recherches à l'Inserm. Cnrs UPR 6520 , Inserm U. 184, faculté de médecine, 11 , rue Humann, 67085 Strasbourg Cedex, France.

\section{TIRÉS A PART}

J.-M. Egly.

\title{
BTF2/TFIIH, un facteur entre transcription et réparation impliqué dans des maladies de la réparation de l'ADN
}

Le xeroderma pigmentosum, le syndrome de Cockayne ou la trichothiodystrophie sont des maladies de la réparation de l'ADN associées à une symptomatologie complexe. La mise en évidence d'une réparation préférentielle des régions transcrites du génome a montré qu'il existe un lien entre la transcription et la réparation qui pourrait être constitué par des effecteurs communs. Or, le facteur BTF2(TFIIH), préalablement identifié comme un acteur indispensable de la réaction de transcription, possède au moins trois sous-unités, ERCC2, ERCC3 et un analogue de SSL1, impliquées dans la réaction de réparation.

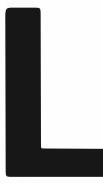

e matériel génétique est perpétuellement soumis à l'irifluence d'agents environnants qui conditionnent les voies d'évolution et de conservation des espèces. L'effet de radiations ionisantes ou d'agents mutagènes peut se traduire par de subtiles modifications de certaines parties du génome entraînant la non-expression du ou des gènes mutés ou la production de protéines altérées dont la fonction première aura été modifiée, perturbant ainsi les processus cellulaires. Les cellules se protègent des conséquences mutagènes de telles altérations, soit en se suicidant si les dommages sont trop importants (apoptose), soit en réparant les régions de l'ADN affectées. Ainsi, pour éliminer des lésions carcinogènes suscitées par des irradia- tions aux ultraviolets ou par différents mutagènes, la cellule mettra en place un mécanisme de réparation comme le système d'excision-resynthèse de nucléotides (NER, pour nucleotide excision-repair) [1]. Une fois l'ADN des gènes réparé, la transcription pourra se poursuivre de façon normale, établissant une relation potentielle entre la réparation des gènes endommagés et la synthèse des ARN messagers correspondants, c'est-à-dire la transcription.

\section{La réparation de I'ADN par le système d'excision- resynthèse de nucléotides}

De nombreux agents chimiques et des types variés de radiations ionisantes sont à l'origine de nombreuses lésions de l'ADN allant de la 


\section{RÉFÉRENCES}

1. Weeda G, Hoeijmakers JHJ, Bootsma D. Genes controlling nucleotide excision repair in eukaryotic cells. BioEssays 1993 ; $15: 249-58$.

2. van Houten B. Nucleotide excision repair in Escherichia coli. Microbiol Rev 1990 ; 54:18-51.

3. Tomkinson AE, Bardwell AJ, Bardwell L, Tappe N], Friedberg EC. Yeast DNA repair and recombination proteins $\operatorname{Rad} 1$ and Rad 10 constitute a single-stranded-DNA endonuclease. Nature $1993 ; 362 ; 860-2$.

4. Habraken Y, Sung P, Prakash L, Prakash S. Yeast excision repair gene $R A D 2$ encodes a single-stranded DNA endonuclease. Nature 1993 ; $366: 365-8$.

5. Cleaver JE, Kraemer KH. Xeroderma pigmentosum. In : Scriver CR, Beaudet AL, Sly WS, Valle D, eds. The Metabolic basis of inherited disease. New York : McGraw-Hill, 1988 : 2949-71.

6. Stefanini M, Lagomarsini P, Giliani S, Tiziana N, Botta E, Peserico A, Kleijer WJ, Lehmann AR, Sarasin A. Genetic heterogeneity of the excision repair defect associated with trichothiodystrophy. Carcinogenesis $1993 ; 14: 1101-5$.

7. Hanawalt $P$, Mellon I. Stranded in an active gene. Curr Biol 1993 ; 3 : 67-9.

8. Selby CP, Sancar A. Molecular mecanism of transcription repair coupling. Science 1993 ; $260: 53-8$.

9. Troelsta C, van Gool A, de Wit J, Vermeulen W, Bootsma D, Hoeijmakers JHJ. ERCC6, a member of a subfamilly of putative helicases, is involved in Cockayne's syndrome and preferential repair of active genes. Cell 1992 ; 71 : 939-53

10. Schaeffer L, Roy R, Humbert S, Moncollin V, Vermeulen W, Hoeijmakers, Chambon P, Egly JM. DNA repair helicase : a component of BTF2(TFIIH) basic transcription factor. Science $1993 ; 260: 58-63$.

11. Feaver WJ, Svejstrup JQ Bardwell L, Bardwell AJ, Buratowsky $\mathbf{S}$, Gulyas KD, Donahue TF, Friedberg EC, Kornberg RD. Dual roles of a multiprotein complex from $\mathrm{S}$. cerevisiae in transcription and DNA repair. Cell 1993 ; $75: 1379-87$.

12. Gerard M, Fisher L, Moncollin V, Chipoulet JM, Chambon P, Egly JM. Purification and interaction properties of the human RNA polymerase $\mathrm{B}$ (II) general transcription factor BTF2. I Biol Chem

simple mutation ponctuelle à de grandes aberrations chromosomiques. Ces modifications du génome peuvent entraîner la mort des cellules ou leur entrée dans le processus de carcinogenèse. Le système NER est le principal mécanisme développé par les cellules pour lutter contre les dommages de l'ADN ; il permet de réparer une gamme extraordinairement vaste de lésions comprenant les différents photoproduits induits par les rayonnements ultraviolets (dimères de thymine, liaisons covalentes intra et intercaténaires...). Ce mécanisme comporte quatre étapes faisant intervenir plusieurs protéines dont certaines activités sont caractérisées (figure 1) : (1) reconnaissance de la lésion; (2) incision et excision du brin endommagé ; (3) resynthèse de ce fragment et (4) ligation du brin néosynthétisé à la matrice.

Chez $E$. coli, ce système est maintenant relativement bien caractérisé [2] et fonctionne de la façon suivante. Un complexe à activité hélicase, composé de deux molécules de la protéine UvrA et d'une molécule de la protéine UvrB (UvrA2B), se déplace le long de l'ADN et positionne UvrB sur les lésions qu'il rencontre. Le complexe, formé par la fixation de UvrB à la lésion d'ADN, est ensuite reconnu par l'endonucléase UvrC qui incise le brin muté de part et d'autre de la lésion. Dans un second temps, l'excision de l'oligonucléotide endommagé, vraisemblablement catalysée par l'activité hélicase de la protéine UvrD, permettra à l'ADN polymérase I de resynthétiser un nouveau fragment qui remplacera le fragment endommagé après action d'une ligase.

Chez les eucaryotes, le système NER semble fonctionner selon le même schéma que chez les bactéries, tout en étant beaucoup plus complexe ; le nombre et la nature des différents partenaires moléculaires mis en œuvre sont encore mal connus. Des études, menées en parallèle sur la levure et sur des cellules de rongeur, permettent de mieux appréhender la complexité de ce mécanisme. Chez la levure, il existe au moins douze souches distinctes déficientes dans le système NER, suggérant l'existence d'au moins douze gènes dont les produits participent au bon déroulement de la réaction de réparation. Il a été démontré que les produits des gènes $R A D 1, R A D 2$, $R A D 3, R A D 4, R A D 10, R A D 14$ et $R A D 25$, dont certains sont à présent clonés, sont absolument indispensables à la réparation, et interviendraient antérieurement à, ou au niveau de, l'étape d'incision. Dans cette optique, certaines activités enzymatiques nécessaires à l'élimination du brin d'ADN endommagé ont été caractérisées. Il a pu être établi que les produits des gènes $R A D 1$ et $R A D 10$, lorsqu'ils sont associés, sont dotés d'une activité endonucléasique [3], activité également exhibée par le produit du gène RAD2 [4]. De même, les produits des gènes $R A D 3$ et $R A D 25$ possèdent une activité hélicase suggérant leur rôle potentiel dans la reconnaissance et ou l'élimination de l'oligonucléotide endommagé.

Par mutagenèse généralisée sur des cellules de hamster ou de souris, plusieurs lignées cellulaires mutantes, sensibles au rayons UV et aux agents génotoxiques, ont été établies [1]. Des tests de complémentation par fusion de cellules ont permis d'identifier au moins onze groupes de complémentation distincts. Les cellules appartenant aux cing premiers groupes sont extrêmement sensibles aux rayons ultraviolets, à la suite de la perturbation d'une étape précédant l'excision. Les diverses souches de cellules de hamster (CHO) sensibles aux ultraviolets, ainsi engendrées, ont été utilisées pour identifier les gènes humains capables de réamorcer cette réaction [1]. Ces gènes ont été dénommés ERCC1 à ERCC7 (pour excision repair cross complementarity).

La mutation des gènes impliqués dans la réparation est à l'origine de maladies autosomiques récessives dénommées syndromes de la réparation, ou réparatoses : xeroderma pigmentosum, le syndrome de Cockayne et la trichothiodystrophie. Xeroderma pigmentosum (XP) est une maladie génétique récessive rare, dans laquelle les patients sont affectés d'une très grande photosensibilité aux ultraviolets, d'une pigmentation anormale des parties de la peau exposées au soleil, et présentent des risques deux mille fois plus élevés que la normale de développer 


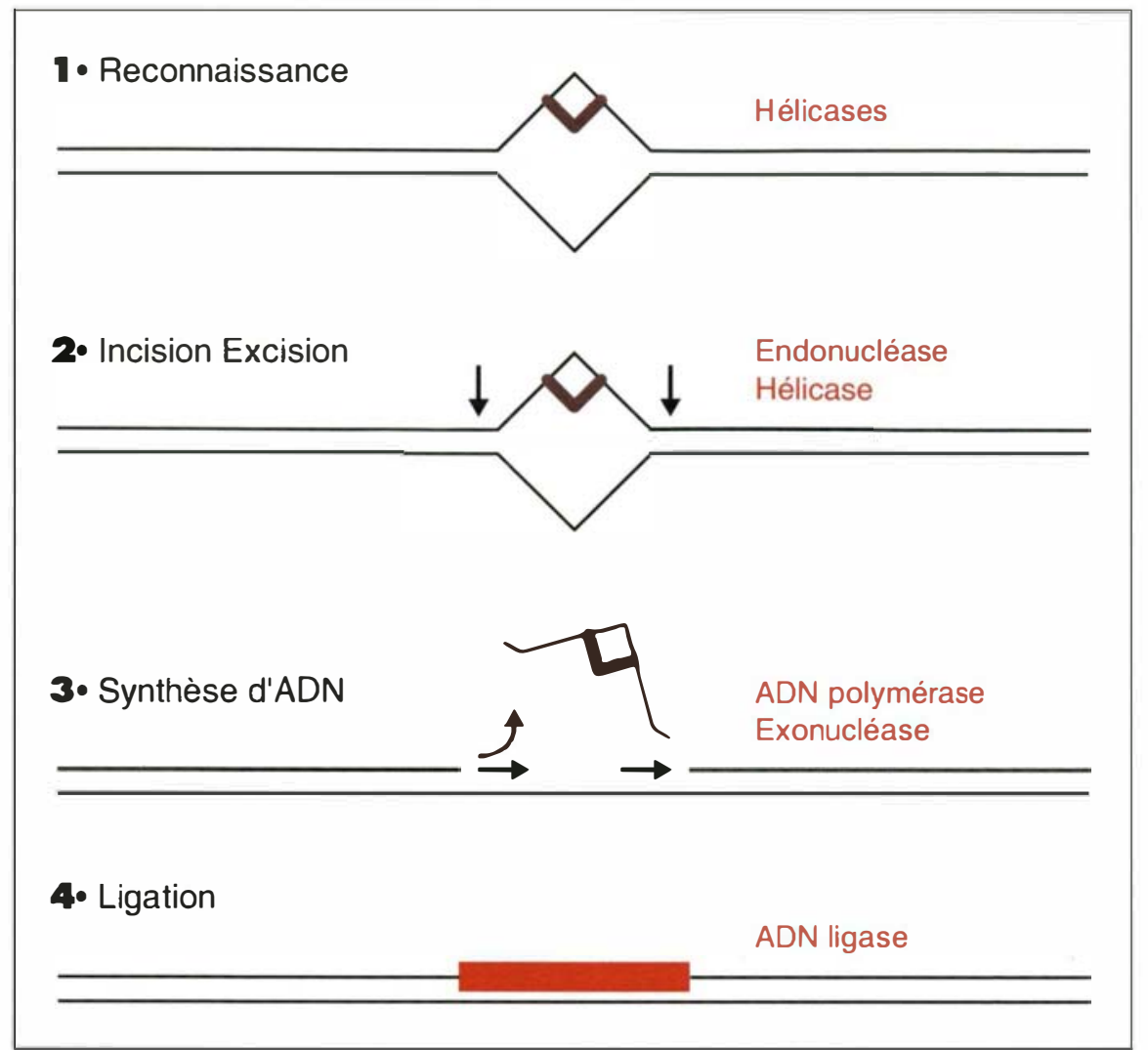

Figure 1. Mécanisme de réparation par le système d'excision-resynthèse de nucléotide (NER). (1) reconnaissance de la lésion; (2) incision et excision du brin endommagé ; (3) resynthèse de ce fragment et (4) ligation du brin néosynthétisé à la matrice.

des tumeurs cutanées. Il faut également noter que les patients déficients en l'un ou l'autre de ces gènes possèdent, dans $20 \%$ à $30 \%$ des cas, des symptômes de dégénérescence neurologique progressive. Sept groupes de complémentation distincts ont été identifiés pour cette maladie : XP-A à XP-G. Un autre groupe, communément appelé XP variant, concerne des patients chez lesquels la réparation de l'ADN se fait par recombinaison ou voie postréplicative [5] ; les patients classés dans ce groupe sont peu sensibles aux ultraviolets. Les patients atteints du syndrome de Cockayne (CS) présentent également une hyper-photosensibilité aux ultraviolets, avec moins de risques de développer des tumeurs cutanées. Ils sont, en revanche, sévèrement retardés sur le plan mental et physique. Deux groupes de complémentation dis$\mathrm{m} / \mathrm{s} n^{\circ} 10$, vol. 10 , octobre 94 gène actif [7]. Cette réparation préferentielle semble être dépendante de l'activité transcriptionnelle de I'ARN polymérase II. Les dimères de thymine engendrés par les rayonnements ultraviolets peuvent, en effet, bloquer le processus d'élongation des transcrits par l'ARN polymérase II. Ainsi, un blocage de la transcription entraine un blocage de la réparation [7]. Ces divers résultats suggèrent l'existence d'un lien direct entre transcription et réparation, concrétisé par un facteur, ou un ensemble de facteurs, pouvant être impliqué dans les deux mécanismes. Chez $E$. coli, le facteur de couplage TRCF (transcription repair coupling factor), produit du gène mfd (mutation frequency decline), est un excellent candidat à cette fonction. Il interagit avec l'ARN polymérase lorsque cette dernière bute sur une lésion, et pourrait, dans un second temps, recruter le facteur UvrA, point de départ de la réaction de réparation [8].

Chez les eucaryotes, les liens entre transcription et réparation semblent être beaucoup plus complexes et ne sont sans doute pas le fait d'une seule protéine. La réponse à cette question devra attendre la caractérisation des différents composants qui sont impliqués dans chaque mécanisme. Cependant, on a pu constater que les cellules appartenant au groupe de complémentation CS-B [9], dont le gène ERCC6 est défectueux, sont uniquement déficientes dans la voie de réparation préférentielle du brin transcrit et non dans la voie générale. ERCC6, qui code pour une protéine dont la structure primaire plaide en faveur d'une activité hélicase, appartient à la famille des protéines SNF2-like, activateurs en puissance de la transcription. Ainsi, l'action de ERCC6, facilitée par le fait que la structure chromatinienne entourant les gènes actifs est beaucoup plus lâche que celle des gènes inactifs, autoriserait une meilleure accessibilité de la matrice d'ADN aux facteurs de réparation. Cependant, ERCC6 n'est certainement pas seul responsable du couplage réparation-transcription ; en effet, les cellules du groupe de complémentation CS-A présentent également des défauts dans leurs systèmes de réparation préférentielle alors 


\section{RÉFÉRENCES}

13. Lu H, Zawel L, Fisher L, Egly JM Reinberg D. Human general transcription factor IIH phosphorylates the C-terminal domain of RNA polymerase II. Nature $1992 ; 358: 641-5$.

14. Schaeffer L, Moncollin V, Roy R, Staub A, Mezzina M, Sarasin A, Weeda G Hoeijmakers JHJ, Egly, JM. The ERCC2/DNA repair protein is associated with the class II BTF2/TFIIH transcription factor. $E M B O J 1994$ (sous presse).

15. Park E, Guzder S, Koken MHM Jaspers-Dekker I, Weeda G, Hoeijmakers JH], Prakash S, Prakash I. RAD25 (SSL2), a yeast homolog of the human xeroderma pigmentosum group $B$ DNA repair gene is essential for viability. Proc Natl Acad Sci USA $1992 ; 89: 11416-20$.

16. Sung P, Bailly V, Weber C, Thompson LH, Prakash L, Prakash S. Human xeroderma pigmentosum group $D$ gene encodes a ma pigmentosum group D gene encodes
DNA helicase. Nature $1993 ; 365: 852-5$.

17. Naumovski L, Chu G, Berg P, Friedberg EC. RAD3 gene of Saccharomyces cerevisiae : nucleotide sequence of wild type and mutant alleles, transcript mapping, and aspects of gene regulation. Mol Cell Biol $1985 ; 5: 17-26$.

18. Sung P, Higgins D, Prakash L, Prakash $S$. Mutation of lysine -48 to arginine in the yeast rad3 protein abolishes its ATPase and helicase activities but not the ability to bind ATP. EMBO J $1988 ; 7$ : 3263-9.

19. Fisher L, Gerard M, Chalut C, Lutz X, Humbert S, Kanno M, Chambon P, Egly JM. Cloning of the 62-kilodalton component of basic transcription factor BTF2 nent of basic transcription
Science $1992 ; 257: 1392-4$.

20. Gileadi O, Feaver WJ, Kornberg RD. Cloning of a subunit of yeast RNA polymerase II transcription factor $b$ and CTD kinase. Science 1992 ; 257 : 1389-92.

21. Humbert S, van Vuuren $H$, Lutz $Y$ Hoeijmakers JHJ, Egly JM, Moncollin V. p44 and p34 subunits of the BTF2/TFIIH transcription factor have homologies with SSLl, a yeast protein involved in DNA repair. $E M B O J 1994$ (sous presse).

22. van Vuuren AJ, Vermeulen W, Ma L Weeda G, Appeldoorn E, Jaspers NGJ, van der Eb AJ, Bootsma D, Humbert $S$ Schaeffer L, Egly JM, Hoeijmakers JHJ Correction of xeroderma pigmentosum repair defect by basal transcription factor BTF2(TFIIH). EMBO J 1994 (sous presse).

23. Wang J, Grossman L. Mutations in the helix-turn-helix motif of the Escherichia col UvrA protein eliminate its specificity for UV-damaged DNA. I Biol Chem 1993 ; 268 : 5323-31.

24. Sarasin A. Les gènes humains de la réparation de l'ADN. médecine/sciences que leur gène ERCC6 est fonctionnel [9]. Le produit du gène $E R C C 5 / X P G$, homologue du gène de levure $R A D 2$ qui code pour une endonucléase, est probablement impliqué lui aussi dans la réparation préférentielle de l'ADN transcrit ; en effet, une mutation de ce gène est associée à des symptômes typiques de CS [1].

La découverte récente du facteur BTF2 humain et du facteur b de levure $[10,11]$, impliqués aussi bien dans la transcription de base des gènes codant pour les protéines que dans la réparation de l'ADN, apporte la preuve biochimique d'un couplage direct entre ces deux phénomènes, et permet de mieux comprendre certains symptômes, à ce jour difficilement explicables, des patients atteints de CS ou de TTD ( $\mathrm{m} / \mathrm{s} n^{\circ} 4$, vol. 9, p. 485).

\section{Le facteur BTF2/TFIIH}

Comme cela a déjà été discuté, le système NER implique un plus grand nombre de facteurs chez les eucaryotes que chez les procaryotes, probablement en raison de la plus grande complexité du génome qu'il faut contrôler (hyperspécialisation). Les régions transcrites seront réparées d'abord, alors que les régions non transcrites, c'est-à-dire la plus grande partie du génome, seront réparées moins rapidement [7]. Cela pourrait justifier la concentration particulièrement élevée de facteurs de réparation au niveau des gènes actifs. Cette hypothèse permet de comprendre pourquoi un nombre conséquent de polypeptides impliqués dans le processus de réparation se trouve associé au facteur BTF2/TFIIH dont le rôle dans la transcription a été montré précédemment $\left(m / s n^{\circ} 4\right.$, vol. 9, p. 485) [12].

Le facteur de transcription BTF2/TFIIH et ses analogues, le facteur $\delta$ chez le rat et le facteur b chez la levure, est un complexe multipeptidique composé d'au moins huit sous-unités qui possèdent diverses activités enzymatiques importantes pour le démarrage de la transcription. Une kinase utilise comme substrat la grande sous-unité de l'ARN polymérase II, et notamment son extrémité carboxy-terminale compo- sée d'une séquence riche en acides aminés thréonine et sérine, répétée entre vingt et cinquante fois selon les espèces. Cette phosphorylation favoriserait, soit la mise en place du complexe actif d'initiation de la transcription, soit le passage à l'étape d'élongation [13]. Deux activités hélicase de polarité opposée et utilisant de l'ATP sont également intimement associées à BTF2/TFIIH $[10,14]$. Le positionnement de ce facteur sur le complexe de préinitiation pourrait, comme cela a déjà été démontré chez les procaryotes, permettre la formation du complexe " ouvert " grâce à ses activités hélicase, exprimées par les sous-unités ERCC3 (89 kDa) et ERCC2 $(80 \mathrm{kDa})$.

Le gène ERCC 3 code pour un polypeptide de 782 acides aminés et a $55 \%$ de similitude avec le gène de levure RAD25. Les deux protéines codées par ces gènes possèdent une activité ADN hélicase de polarité 3'5', dépendante d'une activité ATPase qui ne se manif este qu'en présence d'ADN [10]. Des expériences de mutagenèse dirigée ont montré qu'une altération du site de fixation de l'ATP inhibait à la fois l'activité ATPase et l'activité hélicase, entraînant la mort de la cellule [15]. Le gène $E R C C 2$ code pour une protéine de 760 acides aminés qui complémente le groupe XP/D et a plus de $50 \%$ de similitude avec le produit du gène $R A D 3$ de levure [1]. Ces deux protéines possèdent également une activité hélicase dépendante de l'ATP et agissent avec une polarité 5'-3' [14, 16]. Des mutations dans Rad3 entraînent l'inhibition de ces activités enzymatiques, et affectent la viabilité de la cellule [17]. Il semble, cependant, que cette viabilité ne nécessite pas que ERCC2 possède une activité hélicase dépendante de l'ATP intacte [18]. L'addition de ERCC2 à un système de transcription in vitro, contenant BTF2 privé de sa sous-unité ERCC2/p80, entraîne une stimulation de la transcription de base [14], démontrant que ERCC2 agit bien sur la transcription. Les rôles respectifs de ERCC2 et ERCC3 dans les activités du complexe BTF2/TFIIH ne sont pas connus.

Les protéines p62 chez l'homme et Tfbl chez la levure ont été les pre- 
mières sous-unitês de BTF2/TFIIH et du facteur b à voir leurs ADNc clonés [19, 20]. I.eur similitude est faible, localisée dans la région médiane de la protéine. Des anticorps dirigés contre ces sous-unités sont capables d'inhiber aussi bien la réaction de transcription que la réaction de réparation, mais on ne dispose d'aucun renseignement sur leur fonction précise. Cependant, on a pu identifier chez la levure des mutants de Tfb 1 sensibles aux ultraviolets, suggérant un rôle spécifique de cette protéine dans le mécanisme de réparation de l'ADN (S. Buratowski, communication personnelle).

Le récent clonage de l'ADNc de la sous-unité p44 de BTF2/TFIII a révélé qu'il s'agissait de l'homologue humain de SSL.1 [21], une protéine de levure impliquée dans la résistance aux ultraviolets et sous-unité du facteur b. Mis à part le fait que ces deux protéines contiennent des structures protéiques en doigts de zinc, et se fixent sans doute à l'AI)N, leur rôle dans la réparation de l'ADN est encore incommu.
Enfin, l'ADNc de la sous-unité p34 de BTF2/TFIIH a été cloné lui aussi et l'analyse de la séquence de cette protéine a révélé une région de forte homologie avec SSL.1, notamment dans la région où il y aurait un motif du type doigt de gant, que l'on trouve également dans p44. Il ne serait donc pas surprenant que cette autre sous-unité du facteur de transcription BTF2/TFIII fût aussi impliquée dans la réparation de l'ADN.

\section{Un complexe multifonctionnel de transcription et de réparation}

En utilisant des anticorps dirigés contre les différents composants de BTF2/TFIIH, il a été possible de dépléter un système acellulaire de réparation et d'inhiber la réaction de transcription in vitro. Lorsqu'ils sont micro-injectés dans des cellules, ces anticorps sont capables d'inhiber aussi bien la transcription que la réparation [22]. Cela, ajouté au fait que des fractions hautement puri-

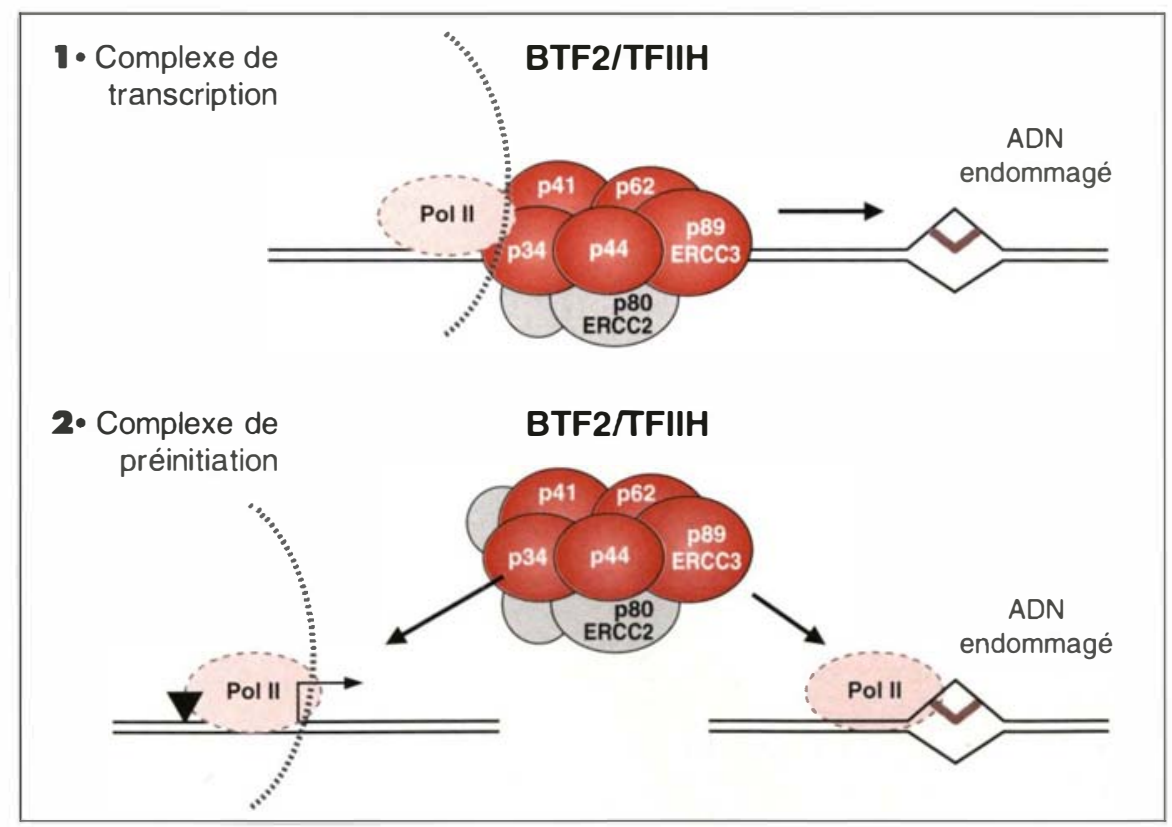

Figure 2. Les modes d'intervention de BTF2/TFIIH dans la transcription et la réparation peuvent suivre deux schémas. BTF2 intègre le complexe d'initiation de la transcription et, soit (1): il reste associé à l'ARN polymérase durant l'élongation et, lorsqu'il rencontre une lésion sur le brin transcrit, un réarrangement du complexe s'opère, permettant l'arrivée de facteurs de réparation, soit (2) : il quitte le complexe après l'initiation et ne rejoint I'ARN polymérase que lorsque celle-ci est arrêtée par une lésion, pour amorcer, en concertation avec $d^{\prime}$ autres facteurs, la réaction de réparation. fiées de $\mathrm{BTF} 2 / \mathrm{TFIIH}$ restaurent la réparation lorsqu'elles sont microinjectées dans des cellules déficientes en ERC.C.2 ou ERCC.3, plaide incontestablement en faveur de la participation directe ou indirecte de chacun des composants identifiés de BTF2/TFIIH dans les réactions de transcription et de réparation [22].

Un objectif commun des systèmes de transcription et de réparation est d'accéder à la matrice d'ADN pour la lire. Cette tâche pourrait être en partie assurée par le complexe multiprotéique BTF2/TFIIH qui possède plusieurs activités. Une activité kinase, capable de phosphoryler l'extrémité carboxy-terminale de la grosse sous-unité de I'ARN polymérase Il, pourrait stimuler l'étape d'initiation et/ou d'élongation de la transcription [13]. Une activité ATPase dépendante de l'ADN, intimement associée à deux activités hélicase, permettrait le passage du complexe de transcription fermé (inactif) au complexe ouvert (actif). Au niveau de la réparation, ces hélicases pourraient signaler la lésion et dérouler la double hélice pour préparer l'excision du brin endommagé (voir ci-après). Les deux sous-unités, qui possèdent des régions susceptibles de former des doigts de zinc, pourraient servir de point d'ancrage de BTF2/TFIIH sur l'ADN et, par la suite, de cible pour d'autres facteurs de transcription et/ou des facteurs de réparation [21].

Dans la réparation par le système NER, des activités hélicase semblent nécessaires lors de deux étapes au moins, la reconnaissance et l'excision. ERC:C:2, ERC:C:3 ou leurs homologues de levure semblent ne pas intervenir dans la réparation après l'étape d'incision, et la récente caractérisation biochimique de ces hélicases peut laisser supposer qu'elles sont impliquées dans la recommaissance des lésions. En effet, ces hélicases présentent des similitudes avec celles du complexe UvrA2B d'E. coli. BTF2/TFIIH, le complexe b de levure et UvrA2B sont tous trois des ensembles multimoléculaires dotés d'activité hélicase. La protéine Rad3, homologue de ERC:C:2 che\% la levure, possède une forte affinité pour les lésions de l'ADN et semble s'y fixer, tout comme UvrB. Enfin, les hélicases 
UvrA et ERC:C'3 contiennent toutes deux une structure boucle-hélice dont l'importance pour la reconnaissance des lésions a été montrée pour UvrA [23]. Par ailleurs, les diverses activités des sous-unités du facteur BTF2/TFIIH, impliqué aussi bien dans la réaction de transcription que dans celle de la réparation, pourraient expliquer l'hétérogénéité des symptômes cliniques observés chez des patients atteints de XP, (SS et TTI) [24]. Des mutations dans l'un des gènes codant pour les sous-unités de BTF2/TFIIH peuvent conférer aux malades les symptômes de CS, en relation avec une perte de la réparation préférentielle des gènes exprimés. Ces mutations, si elles n'entraînent pas la mort de la cellule, pourraient aussi perturber la transcription spécifique de certains gènes, soit en inhibant la fonction de la sous-unité, produit du gène muté, soit en perturbant l'interaction de cette sous-unité avec un facteur de régulation différent ou avec des séquences spécifiques d'ADN. Certains signes cliniques seront alors la conséquence secondaire des anomalies d'expression de gènes dépendant particulièrement de certaines activités du complexe BTF2/TFIIH.

Quoi qu'il en soit, l'association d'anomalies de la réparation de l'AIN à des perturbations des fonctions transcriptionnelles de BTF2/ TFIIH pourrait permettre d'expliquer la symptomatologie de maladies génétiques aux mécanismes encore obscurs.

De nombreuses questions restent non résolues. On suppose que la présence de dimères de thymine bloque l'ARN polymérase II engagée dans le processus d'élongation. L'immobilisation d'un tel complexe permettrait à BTF2/TFIIH d'attirer les autres facteurs de réparation. Cependant, la présence de BTF2/TFIIH au niveau du complexe d'élongation n'a absolument pas été démontrée à ce jour. Une autre possibilité serait que $\mathrm{BTF} 2$ reconnaisse uniquement l'ARN polymérase "à l'arrêt", soit au niveau du site de démarrage, soit bloquée par une lésion (figure 2).

L'immobilisation de l'ARN polymérase sur l'ADN a des effets négatifs, non seulement sur la transcription, réplication [7]. En effet, après irradiation par les ultraviolets, les cellules issues de malades atteints du C.S sont incapables de retrouver un niveau normal de synthèse, non seulement d'ARN (transcription), mais aussi d'ADN (réplication), alors qu'elles sont capables de réparer les lésions dans les régions non transcrites du génome. Puisque les étapes finales du système NER impliquent une resynthèse du fragment excisé, il est facile d'imaginer une relation étroite entre transcription, réparation et réplication. Cette hypothèse est d'autant plus séduisante que BTF2/TFIIH possède des activités (hélicase et de liaison à l'ADN) prêtes à jouer un rôle dans ces trois réactions. Un défaut de BTF2/TFIIH dans l'exercice de sa fonction de transcription ne pourrait-il pas être à l'origine de diverses maladies génétiques difficilement expliquées à ce jour, les syndromes de transcription?

\section{Summary}

BTF2/TFIIH, a factor with mixed transcription and reparation activities, is involved in DNA reparation diseases

The nucleotide excision repair (NER) pathway is one of the principal pathways in cell of eliminating a wide variety of unrelated lesions in a complex multistep reaction. Because of the effects of lesions produced by genotoxic agents on the vital transcription process, their removal by NER must occur in a way closely coordinated with transcription. The dual role of BTF2/TFIIH in transcription and repair may explain the heterogeneity of symptoms that, at first glance, seem completely unrelated to skin UV photosensitivity, such as developmental retardation, brittle hair and nails, ichthyosis, deafness, and neurodysmyelination observed in the XP, ('S and TTI) disorders. 